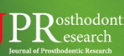 \\ Journal of Prosthodontic Research
}

\author{
Official Journal of Japan Prosthodontic Society
}

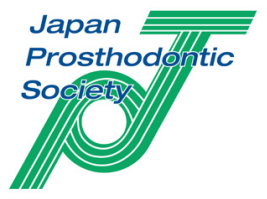

Original article

\section{Effects of dimensions of laser-milled grid-like microslits on shear bond strength between porcelain or indirect composite resin and zirconia}

\author{
Shogo Iwaguro ${ }^{a}$, Saiji Shimoe ${ }^{\text {b,* }}$, Hiroto Takenaka ${ }^{\mathrm{c}}$, Yuki Wakabayashi ${ }^{\mathrm{d}}$, Tzu-Yu Peng ${ }^{\mathrm{e}}$, Masato Kaku ${ }^{\mathrm{b}}$ \\ ${ }^{a}$ Division of Dental Technician, Department of Clinical Practice and Support, Hiroshima University Hospital, Hiroshima, Japan. \\ ${ }^{b}$ Department of Anatomy and Functional Restorations, Integrated Health Sciences, Hiroshima University Graduate School of Biomedical \& Health Sciences, Hiroshima, \\ Japan. \\ ${ }^{c}$ Dental Laboratory Center, Department of Medical Technology, Nagasaki University Hospital, Nagasaki, Japan \\ ${ }^{d}$ Department of Dental Medical Laboratory, Hokkaido University Hospital, Sapporo, Hokkaido, Japan \\ ${ }^{e}$ School of Dentistry, College of Oral Medicine, Taipei Medical University, Taipei, Taiwan
}

\begin{abstract}
Purpose: Zirconia cores and frameworks are widely used in restorative dentistry. Although these structures are veneered with porcelain for esthetic reasons, the use of indirect composite resins (ICRs) is expected to increase in the future. The purpose of this study was to investigate the effects of microslits of different dimensions formed by $\mathrm{Nd}: \mathrm{YVO}_{4}$ laser machining on the bond strength between two types of zirconia ( 3 mol\% yttria-partially stabilized zirconia (Y-TZP) and ceria-partially stabilized zirconia/alumina nanocomposite (Ce-TZP/A)) and porcelain or an ICR.

Methods: The zirconia disks were assigned as follows: 1) blasted with alumina particles (AB) and 2-4) surface machined with gridded microslits with a width, pitch, and depth of 50,75, or $100 \mu \mathrm{m}$ (MS50, MS75, and MS100, respectively). After the bonding of the veneering materials to the disks, half of the specimens veneered with the ICR were subjected to thermocycling $\left(4-60^{\circ} \mathrm{C}, 20000\right.$ cycles). All the specimens were subsequently shear tested ( $\left.\mathrm{n}=10 / \mathrm{group}\right)$.

Results: There was no significant difference between the groups of the disks bonded to porcelain. On the other hand, for the disks bonded to the ICR, the bond strengths of the MS groups after thermocycling were statistically higher than that of the AB group. However, there was no significant difference in the bond strengths of the disks with different microslits.

Conclusions: Within the study limitations, it can be concluded that, for porcelain, the design of the mechanical retentive structure must be modified. However, for the investigated ICR, a simple gridded pattern can improve the bond strength with zirconia.
\end{abstract}

Keywords: Zirconia, Porcelain, Indirect composite resin, Laser machining, Microslits

Received 18 August 2020, Accepted 3 March 2021, Available online 29 July 2021

\section{Introduction}

Zirconia has several advantages, including an esthetically pleasing color, high biocompatibility, and remarkable mechanical properties [1-6]. In restorative dentistry, zirconia restorations are employed as an alternative to metals, for example, inlay, monolithic anatomically contoured crown, and core materials for ceramic crowns. However, monolithic zirconia crowns have limitations in terms of the matching of the individual tooth color, although more translucent zirconia systems have been developed. This is because current zirconia processing technologies cannot mimic the internal structure of individual teeth. For patients desiring highly esthetic restorations, prostheses consisting of porcelain fused to zirconia are usually used because of their wider range of shades. In the cases where the veneered porcelain on zirconia has been chipped, it may be preferable to

\footnotetext{
* Corresponding author at: Department of Anatomy and Functional Restorations, Integrated Health Sciences, Hiroshima University Graduate School of Biomedical \& Health Sciences, 2-3 Kasumi 1-chome, Minami-ku, Hiroshima 734-8553, Japan.

E-mail address: shimoe@hiroshima-u.ac.jp (S. Shimoe).
}

repair it intraorally, given the time and expense involved in the removal, the cost of remanufacturing, and the burden placed on the patient.

On the other hand, since indirect composite resin also have a wide range of color application and potential for used as veneering material to zirconia. In addition, since composite resin can be utilized to repair the exposed zirconia frame in intraoral repairs, significant efforts have been made to improve zirconia-composite bonding [7-10]. Besides, when using zirconia for a monolithic restoration, a fine polished zirconia can prevent wear the opposing teeth, while a roughened zirconia can damage it [11-13]. Furthermore, a microhybrid composite resin containing a prepolymerized filler prevents the reduction in occlusal vertical dimension and damages of both aesthetic restoration and tooth by minimizing the antagonistic wear [13]. Thus, to increase the safety to the opposing teeth as well as the ease of repairing fractured restorations, prostheses involving the bonding of indirect composite resins to zirconia may find greater use in the future.

To ensure long-term clinical success, the bond strength between zirconia and veneering materials must be high and durable even in an oral cavity environment. Mechanical interlocking is one of the most important methods for ensuring strong adhesion during the bonding of porcelain or indirect composite resins to core materials [14-19]. The procedures for manufacturing zirconia restorations are unsuitable for producing a microstructure that can ensure this. This is because of the limitations 
related to the minimum diameter of the milling burs used for dental computer-aided design/computer-assisted manufacturing (CAD/CAM). Although there are several studies on surface roughening methods for zirconia, such as grinding with diamond bur [20], alumina blasting $[8,10,20-23]$, acid etching [10,24], and forming a porous structure [14], there are few reports on the effects of the machining such as milling [25] of a retentive structure in zirconia for improving the bond strength.

Laser machining is another approach for the surface treatment of zirconia and has been investigated previously. Industrial lasers such as carbon dioxide $\left(\mathrm{CO}_{2}\right)$ [15], neodymium-doped yttrium aluminum garnet (Nd:YAG) [9,16,23,26,27], Er:YAG [9], and erbium chromium:yttrium scandium gallium garnet (Er,Cr:YSGG) [28] lasers have been used previously for the surface treatment on zirconia. It has also been reported that zirconia can be processed using a computer numerical control (CNC) neodymium-doped yttrium orthovanadate $\left(\mathrm{Nd}: \mathrm{YVO}_{4}\right)$ laser $[29,30]$.

There are no data on the characteristics of the bonds formed between zirconia with microstructure by $\mathrm{CNC}$ laser machining of uniform dimensions and other materials. The $\mathrm{CNC} \mathrm{Nd}: \mathrm{YVO}_{4}$ laser has the potential to overcome the disadvantages of bur milling in zirconia core fabrication because it can process and produce more detail than burs [29], CNC laser machines have not yet been developed for dental applications and require larger machinery and processing times compared to alumina blasting and dental lasers. If the size of the machine can be downsized and the processing time can be shortened, it may be used for dental use in the future. We previously investigated the effect of microslits formed on the surface of zirconia, formed by machining using a CNC Nd:YVO laser on the bond strength between two types of zirconia and porcelain or two types of resins [17]. We had found that the CNC laser ablation of zirconia allowed for the formation of regular slit-like microstructures with precision and that these microstructures improved the bond strength and adhesion with the resins. No improvement was observed in case of some combinations of zirconia and the investigated materials even after the formation of the microslits. Hence, we hypothesized that the bond strength between zirconia and porcelain or resins can be improved by varying the design of the machined microslits. Hence, the purpose of this study was to evaluate the effects of three different dimensions of microslits on the bond strength between two types of zirconia and porcelain or an indirect composite resin.

\section{Materials and methods}

\subsection{Specimen fabrication and surface treatment}

The materials used in this study are listed in Table 1. Two types of tetragonal zirconia polycrystals (TZP) were used, namely, $3 \mathrm{~mol} \%$ yttriapartially stabilized zirconia (Y-TZP) and ceria-partially stabilized zirconia/ alumina nanocomposite (Ce-TZP/A). The Y-TZP (TZ-3YSB-E, Tosoh Corp., Tokyo, Japan) and Ce-TZP/A (C-Pro NANO ZR, Yamakin Co., Ltd., Osaka, Japan) samples were sintered for $2 \mathrm{~h}$ at $1450^{\circ} \mathrm{C}$ to produce cylinders with diameters of $10 \mathrm{~mm}$. Then, the cylinders were sliced into disk-shaped specimens with a diameter of $10 \mathrm{~mm}$ and a thickness of $2.5 \mathrm{~mm}$. All the disks were ground flat using a series of silicon carbide abrasive papers (up to 600\# grit). Next, the zirconia disks were randomly assigned to four groups. The first group, denoted AB, was blasted with alumina particles using a grit blaster (Basic Quattro, Renfert GmbH, Hilzingen, Germany). In the case of the other three groups, gridded microslits with a width, pitch and depth of 50, 75, or $100 \mu \mathrm{m}$ (MS50, MS75, and MS100, respectively) were machined on the disk surfaces. Figure 1 shows a schematic drawing of the specimen with the microslits pattern.

The alumina blasting of the $\mathrm{AB}$ group was performed using a $50 \mu \mathrm{m}$ alumina powder (Cobra, Renfert $\mathrm{GmbH}$, Hilzingen, Germany) for $10 \mathrm{~s}$ at a pressure of $0.3 \mathrm{MPa}$. The distance between the blaster nozzle and the disk surface was $10 \mathrm{~mm}$. The microslits formed on the disks in the MS groups were fabricated using a CNC laser machine (LASERTEC 40, DMG MORI, Nagoya, Japan). The laser medium was $\mathrm{Nd}: \mathrm{YVO}_{4}$, and the laser irradiation parameters were as follows: frequency of $70 \mathrm{kHz}$, wavelength of 1,065 $\mathrm{nm}$, distance from the surface of $60 \mathrm{~mm}$, and angle of incidence of $90^{\circ}$. After the completion of the laser machining process, the irradiated surfaces
Table 1. Materials used for this study.

\begin{tabular}{llll}
\hline Material & Main Composition & Manufacturer & Lot No. \\
\hline Zirconia & & $\begin{array}{l}\text { Tosoh Corp., Tokyo, } \\
\text { Japan }\end{array}$ & 18007813 \\
$\begin{array}{l}\text { TZ-3YSB-E (Y-TZP) } \\
\text { C-Pro NANO ZR (Ce- }\end{array}$ & $\mathrm{ZrO}_{2}, \mathrm{Y}_{2} \mathrm{O}_{3}$ & $\begin{array}{l}\text { Yamakin Co., Ltd., } \\
\text { Osaka, Japan }\end{array}$ & CD90011076J \\
$\begin{array}{lll}\mathrm{ZrO}_{2}, \mathrm{Al}_{2} \mathrm{O}_{3}, \mathrm{CeO}_{2} \text { ) } \\
\text { Porcelain }\end{array}$ & \multicolumn{3}{c}{$\begin{array}{l}\text { Shofu Inc., Kyoto, } \\
\text { Japan }\end{array}$} \\
Vintage ZR, & $\begin{array}{l}\text { Aluminosilicate glass, } \\
\text { Glycerin, Propylene } \\
\text { glycol }\end{array}$ & 071596 \\
Opaque Liner A3O & Aluminosilicate glass & 051670 \\
Body A3B & &
\end{tabular}

\begin{tabular}{|c|c|c|c|}
\hline Body A3B & Aluminosilicate glass & & 051670 \\
\hline \multicolumn{4}{|l|}{ Indirect composite resin } \\
\hline Gradia & & $\begin{array}{l}\text { GC Corp., Tokyo, } \\
\text { Japan }\end{array}$ & \\
\hline Foundation opaque & \multirow{3}{*}{ 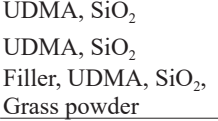 } & & 1510142 \\
\hline Opaque A3 & & & 1511021 \\
\hline Dentin A3 & & & 1501231 \\
\hline \multicolumn{4}{|l|}{ Primer } \\
\hline $\begin{array}{l}\text { Clearfil Ceramic Primer } \\
\text { Plus }\end{array}$ & MDP, ethyl alcohol & $\begin{array}{l}\text { Kuraray Noritake } \\
\text { Dental Inc., Tokyo, } \\
\text { Japan }\end{array}$ & 7R0018 \\
\hline
\end{tabular}
UDMA = urethane dimethacrylate; MDP = 10-methacryloyloxydecyl dihydrogen
phosphate

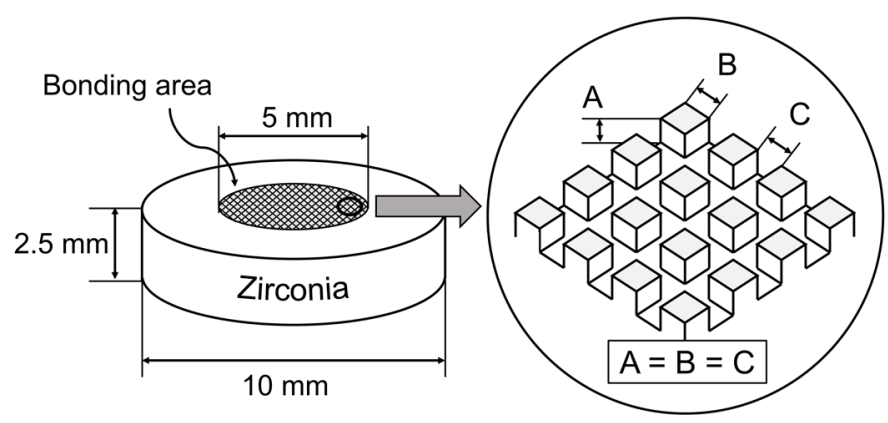

Fig. 1. Schematic drawing of specimens with microslit pattern $(A=B=C)$ with slit dimensions of 50,75 , and $100 \mu \mathrm{m}$.

turned black. Therefore, the specimens were heated at a rate of $55^{\circ} \mathrm{C} / \mathrm{min}$ and held at $1,000^{\circ} \mathrm{C}$ for $5 \mathrm{~min}$ to restore their white color. Figure 2 and 3 show SEM images of surface of Y-TZP and Ce-TZP/A before veneering.

\subsection{Veneering procedure}

The procedure for veneering porcelain on the zirconia disks was accomplished as follows. First, a liner material (Vintage ZR, Shofu Inc., Kyoto, Japan) was applied on the zirconia specimen over the bonding area (circle with a hole with a diameter of $5 \mathrm{~mm}$ ), which was defined using tape. The tape was then removed from the surface, and the specimen was fused in a porcelain furnace (Master S, Denken-Highdental Co., Ltd., Kyoto, Japan) as per the manufacturer's instructions. Next, dentin porcelain (Vintage ZR, Shofu Inc., Japan) was filled in a cylindrical vinyl mold (internal diameter of $6 \mathrm{~mm}$, length of $2.5 \mathrm{~mm}$, and a wall thickness of $1 \mathrm{~mm}$ ) fixed on the zirconia sample. The mold was removed after the completion of the condensation and drying process, and the specimen was fired in a porcelain furnace as per the manufacturer's instructions. Finally, all the specimens $(n=10)$ were immersed in distilled water at $37^{\circ} \mathrm{C}$ for 24 $\mathrm{h}$ and subsequently subjected to shear tests.

The procedure for bonding the zirconia disks with the indirect composite resin was as follows. A double-sided tape with a circular $5 \mathrm{~mm}$ diameter hole was stuck on the surface of the zirconia specimen to define the bonding area. Next, the primer (Clearfil Ceramic Primer Plus, Kuraray Noritake Dental Inc., Tokyo, Japan) was applied to the bonding area. Then, 

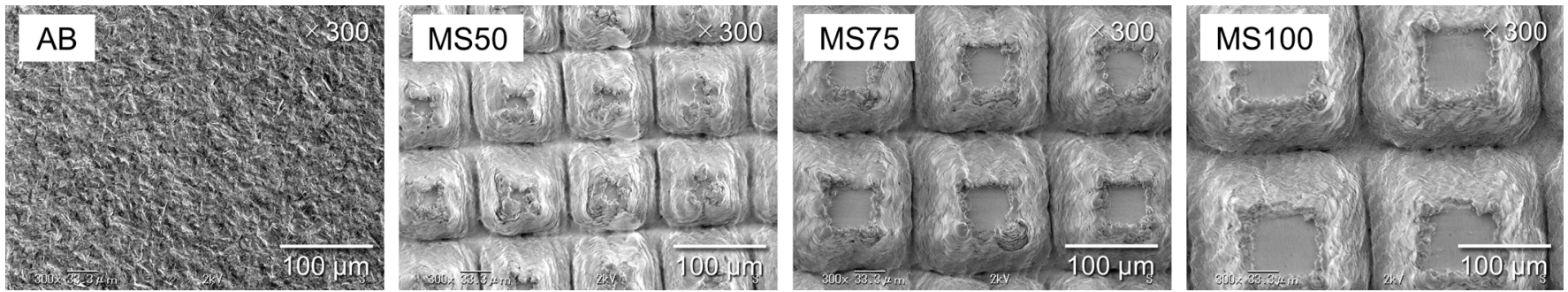

Fig. 2. SEM images of surfaces of Y-TZP samples before veneering (300×).
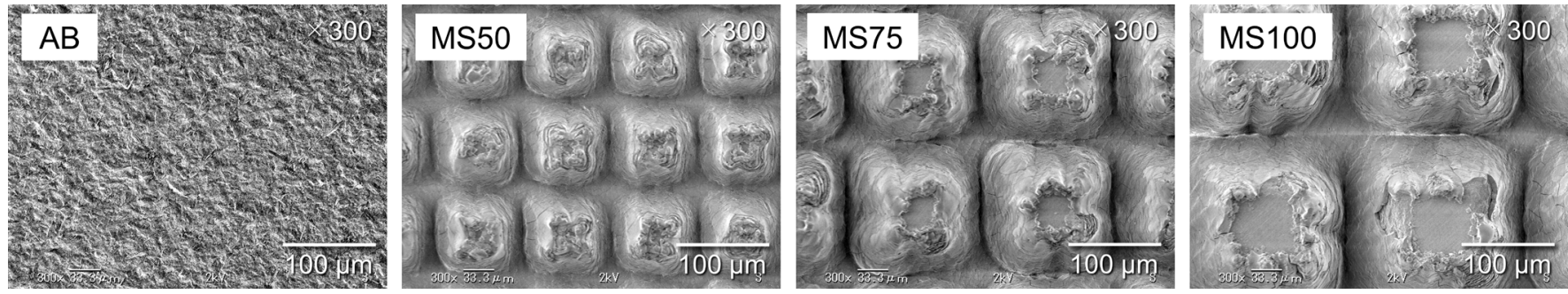

Fig. 3. SEM images of surfaces of Ce-TZP/A samples before veneering (300×).

the foundation opaque and opaque (Gradia, GC Corp., Tokyo, Japan) were applied on the primed surface and photopolymerized for $60 \mathrm{~s}$ in the lightcuring unit (Hi-light Power, Kulzer GmbH, Hanau, Germany). After the completion of the curing process, a brass mold (internal diameter of $6 \mathrm{~mm}$, length of $2 \mathrm{~mm}$, and a wall thickness of $1 \mathrm{~mm}$ ) was fixed using the doublesided tape, and a dentin resin (Gradia, GC) was filled in the mold. Final polymerization was performed for $180 \mathrm{~s}$ in the light-curing unit. All the specimens $(n=20)$ were immersed in distilled water at $37^{\circ} \mathrm{C}$ for $24 \mathrm{~h}$, and this state was defined as "no thermocycling." Half of these specimens (n $=10$ ) were placed in a thermocycling apparatus (Thermal Cycler, Nissin Seiki Co. Ltd., Hiroshima, Japan) and cycled between $4{ }^{\circ} \mathrm{C}$ and $60^{\circ} \mathrm{C}$ in water (dwell time per water bath for $1 \mathrm{~min}$ ) for 20,000 cycles.

\subsection{Shear test and statistical analysis}

The specimens veneered with porcelain or the indirect composite resin were placed in a testing jig, and a shear test was performed at a crosshead speed of $0.5 \mathrm{~mm} / \mathrm{min}$ using a universal testing machine (AGS-5kNJ, Shimadzu, Kyoto, Japan). The shear bond strength (SBS) was calculated by dividing the peak load $(\mathrm{N})$ by the bonding area. Statistical comparisons of the test results for the various groups were performed using the twoway analysis of variance (ANOVA) method and Scheffe's test for multiple comparisons. The statistical significance level was set at 0.05 . In addition, to analyze the effects of the thermocycling, the SBS values for 0 and 20,000 cycles of identical surface treatment were compared using MannWhitney's $U$ test. A $P$ value of less than 0.05 was considered to indicate a significant difference. All the analyses were performed using SPSS 15.0 for Windows (SPSS Japan Inc., Tokyo, Japan).

\subsection{Statistical analysis}

After the shear bond tests, the bond failure type was categorized as one of the following: adhesive failure at the zirconia-veneer material interface (A), cohesive failure within the veneer material $(\mathrm{C})$, or a mixture of adhesive and cohesive failures (AC). The fracture surfaces of the zirconia disks used for the shear bond test were observed using scanning electron microscopy (SEM, SE-8000, Keyence Corp., Osaka, Japan) at a magnification of $500 \times$

\section{Results}

The mean SBS values of the porcelain-on-zirconia specimens along with the standard deviations and statistical significances are listed in Table 2.
The SBS values of the Y-TZP samples ranged from a maximum of 20.5 $\mathrm{MPa}$ (in the case of the MS75 group) to a minimum of $17.3 \mathrm{MPa}$ (in the case of the $\mathrm{AB}$ group). The SBS values of the Ce-TZP/A ranged from a maximum of $19.2 \mathrm{MPa}$ (MS100 group) to a minimum of $17.3 \mathrm{MPa}$ (AB group). However, no statistically significant differences were found between the groups. The failure modes of the various samples after the shear tests are listed in Table 3. All the MS groups showed cohesive failure (C). Further, the SEM observations performed after the shear tests showed that residual porcelain was present in the microslits (Figs. 4 and 5). On the other hand, all the $\mathrm{AB}$ groups exhibited mixed failure (AC).

The mean SBS values of the indirect-composite-resin-on-zirconia samples and their standard deviations and statistical significances are listed in Table 4. In the case of the mean SBS values of the Y-TZP samples before thermocycling, there was no significant difference between the different groups. Similarly, with respect to the mean SBS values of the Ce-TZP/A samples, no statistical difference was found. On the other hand, after thermocycling, the SBS values of both types of zirconia in all the MS groups were significantly higher than those of the $\mathrm{AB}$ groups. The rate of decrease in the SBS after thermocycling in the case of the $\mathrm{AB}$ groups was $46.4 \%$ for Y-TZP and $35.2 \%$ for Ce-TZP/A. In contrast, the MS groups corresponding to the two types of zirconia did not show a significant decrease. Further, with respect to the microslit dimensions, there was no significant difference between the groups that were subjected to thermocycling and those that were not. Table 5 shows the failure modes as determined after the shear tests. Before thermocycling, all the groups corresponding to the two types of zirconia exhibited cohesive failure (C). On the other hand, for both zirconia types, after thermocycling, 80 to $90 \%$ of the AB specimens showed mixed failures (AC) while all the specimens in the MS groups exhibited cohesive failure (C). SEM observations performed after the tests revealed that the microslits in the zirconia specimens (MS50, MS75, and MS100) were filled with the indirect composite resin. This was true both before and after thermocycling (Figs. 6 and 7).

\section{Discussion}

In restorative dentistry, the zirconia cores and frameworks used are veneered with porcelain to tailor them to the needs of individual patients. The veneering porcelain must be applied not only to teeth but also to the gingival area in case of large bone defects, such as those requiring dental implants [31]. Considering the ease of repair and the material properties of indirect composite resins, they can be substituted readily for porcelain in veneering. Irrespective of the material used, strong adhesion and high bond 
Table 2. Shear bond strengths of porcelain veneered to zirconia.

\begin{tabular}{cccc}
\hline Groups & Mean & SD & Category \\
\hline Y-TZP & & & \\
AB & 17.3 & 5.0 & $\mathrm{a}$ \\
MS50 & 19.0 & 4.9 & $\mathrm{a}$ \\
MS75 & 20.5 & 3.1 & $\mathrm{a}$ \\
MS100 & 20.4 & 5.0 & $\mathrm{a}$ \\
Ce-TZP/A & & & \\
AB & 17.3 & 2.8 & $\mathrm{~A}$ \\
MS50 & 17.5 & 3.7 & $\mathrm{~A}$ \\
MS75 & 17.9 & 4.6 & $\mathrm{~A}$ \\
MS100 & 19.2 & 3.9 & $\mathrm{~A}$ \\
\hline
\end{tabular}

SD: Standard deviation; Category: Identical letters indicate that values are not statistically different $(P \geqq 0.05)$; S: Significant difference between pre- and postthermocycling bond strengths $(P<0.05)$.
Table 3. Failure modes of porcelain after shear bond testing.

\begin{tabular}{cccc}
\hline \multicolumn{1}{c}{ Froups } & & & \\
\cline { 2 - 4 } Y-TZP & A & AC & C \\
AB & 3 & 7 & 0 \\
MS50 & 0 & 0 & 10 \\
MS75 & 0 & 0 & 10 \\
MS100 & 0 & 0 & 10 \\
Ce-TZP/A & & & \\
AB & 0 & 10 & 0 \\
MS50 & 0 & 0 & 10 \\
MS75 & 0 & 0 & 10 \\
MS100 & 0 & 0 & 10 \\
\hline
\end{tabular}

A: Adhesive failure at the zirconia-porcelain interface

C: Cohesive failure within the porcelain;

AC: Combination of adhesive and porcelain failures.
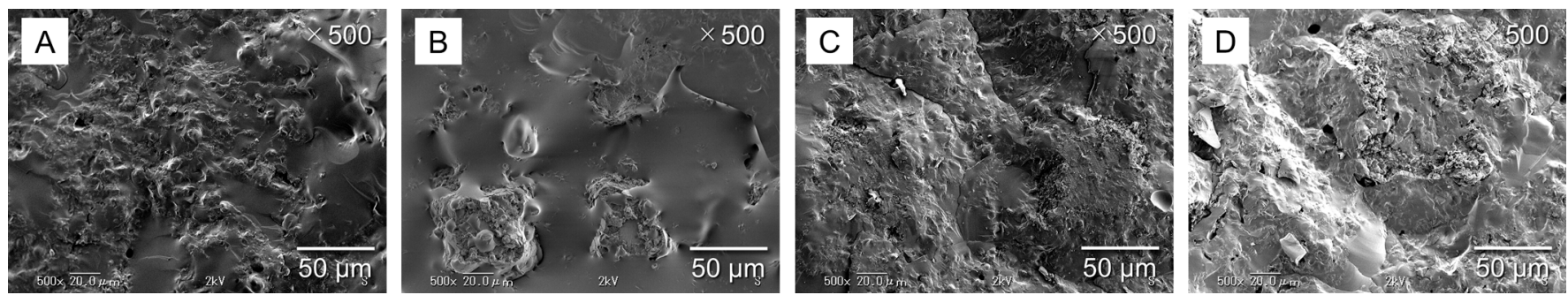

Fig. 4. SEM images of debonded surfaces of Y-TZP samples veneered with porcelain (500×): (A) alumina-blasted specimens and (B-D) specimens with machined microslits with dimensions of 50, 75, and $100 \mu \mathrm{m}$ (MS50, MS75, and MS100, respectively).
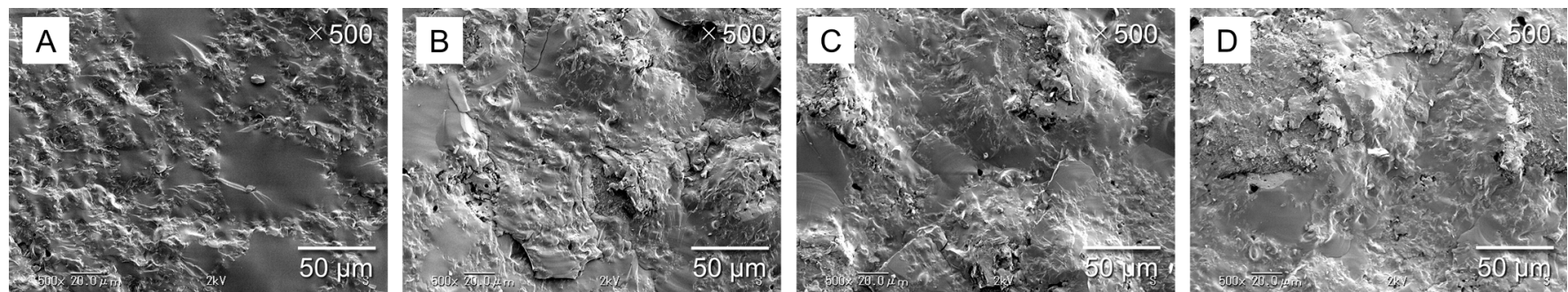

Fig. 5. SEM images of debonded surfaces of Ce-TZP/A samples veneered with porcelain (500×): (A) alumina-blasted specimens and (B-D) specimens with machined microslits with dimensions of 50, 75, and $100 \mu \mathrm{m}$ (MS50, MS75, and MS100, respectively).

Table 4. Shear bond strengths of indirect composite resin veneered to zirconia.

\begin{tabular}{|c|c|c|c|c|c|c|c|c|}
\hline \multirow{2}{*}{ Groups } & \multicolumn{3}{|c|}{0 Thermocycles } & \multicolumn{3}{|c|}{ 20,000 Thermocycles } & \multirow[t]{2}{*}{$\mathrm{S}$} & \multirow[t]{2}{*}{ Reduction } \\
\hline & Mean & SD & Category & Mean & SD & Category & & \\
\hline \multicolumn{9}{|l|}{ Y-TZP } \\
\hline $\mathrm{AB}$ & 28.9 & 3.5 & $\mathrm{a}$ & 15.5 & 4.7 & $\mathrm{~b}$ & $\mathrm{~S}$ & $46.4 \%$ \\
\hline MS50 & 28.6 & 4.5 & a & 33.1 & 7.2 & $\mathrm{c}$ & & $-15.7 \%$ \\
\hline MS75 & 33.5 & 6.0 & a & 31.5 & 6.4 & $\mathrm{c}$ & & $6.0 \%$ \\
\hline MS100 & 29.2 & 2.9 & a & 33.5 & 4.2 & $\mathrm{c}$ & & $-14.7 \%$ \\
\hline \multicolumn{9}{|l|}{$\mathrm{Ce}-\mathrm{TZP} / \mathrm{A}$} \\
\hline $\mathrm{AB}$ & 25.6 & 2.7 & A & 16.6 & 5.9 & B & $\mathrm{S}$ & $35.2 \%$ \\
\hline MS50 & 27.1 & 3.3 & A & 34.5 & 5.4 & C & & $-27.3 \%$ \\
\hline MS75 & 26.6 & 2.9 & A & 32.9 & 5.9 & $\mathrm{C}$ & & $-23.7 \%$ \\
\hline MS100 & 27.6 & 5.1 & A & 32.0 & 3.8 & $\mathrm{C}$ & & $-15.9 \%$ \\
\hline
\end{tabular}

SD: Standard deviation; Category: Identical letters indicate that values are not statistically different $(P \geqq 0.05)$; S: Significant difference between pre- and post-thermocycling bond strengths $(P<0.05)$.
Table 5. Failure modes of indirect composite resin after shear bond testing.

\begin{tabular}{lllllll}
\hline Groups & \multicolumn{3}{c}{0 Thermocycles } & \multicolumn{3}{c}{ 20,000 Thermocycles } \\
\cline { 2 - 7 }$\quad$ Failure mode & A & AC & C & A & AC & C \\
\hline Y-TZP & 0 & 1 & 9 & 1 & 9 & 0 \\
AB & 0 & 0 & 10 & 0 & 0 & 10 \\
MS50 & 0 & 0 & 10 & 0 & 0 & 10 \\
MS75 & 0 & 0 & 10 & 0 & 0 & 10 \\
MS100 & & & & & & \\
Ce-TZP/A & 0 & 0 & 10 & 2 & 8 & 0 \\
AB & 0 & 0 & 10 & 0 & 0 & 10 \\
MS50 & 0 & 0 & 10 & 0 & 0 & 10 \\
MS75 & 0 & 0 & 10 & 0 & 0 & 10 \\
MS100 & 0 & & & & & \\
\hline A A
\end{tabular}

A: Adhesive failure at the zirconia-resin interface;

C: Cohesive failure within the resin;

AC: Combination of adhesive and cohesive failures. 

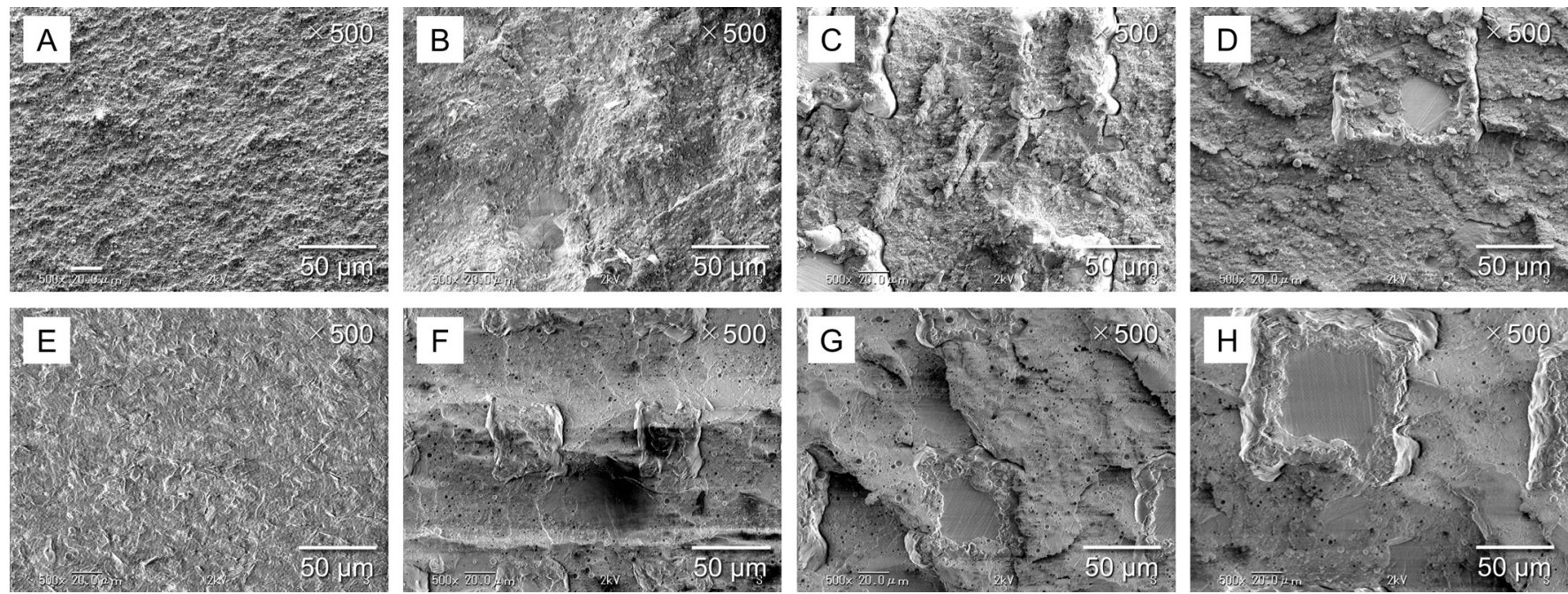

Fig. 6. SEM images of debonded surfaces of Y-TZP samples veneered with indirect composite resin (500×): (A) alumina-blasted specimens and (B-D) specimens with machined microslits with dimensions of 50, 75, and $100 \mu \mathrm{m}$ (MS50, MS75, and MS100, respectively) before thermocycling and (E) alumina-blasted specimens and (F-H) specimens with machined microslits with dimensions of 50, 75, and $100 \mu \mathrm{m}$ (MS50, MS75, and MS100, respectively) after thermocycling.
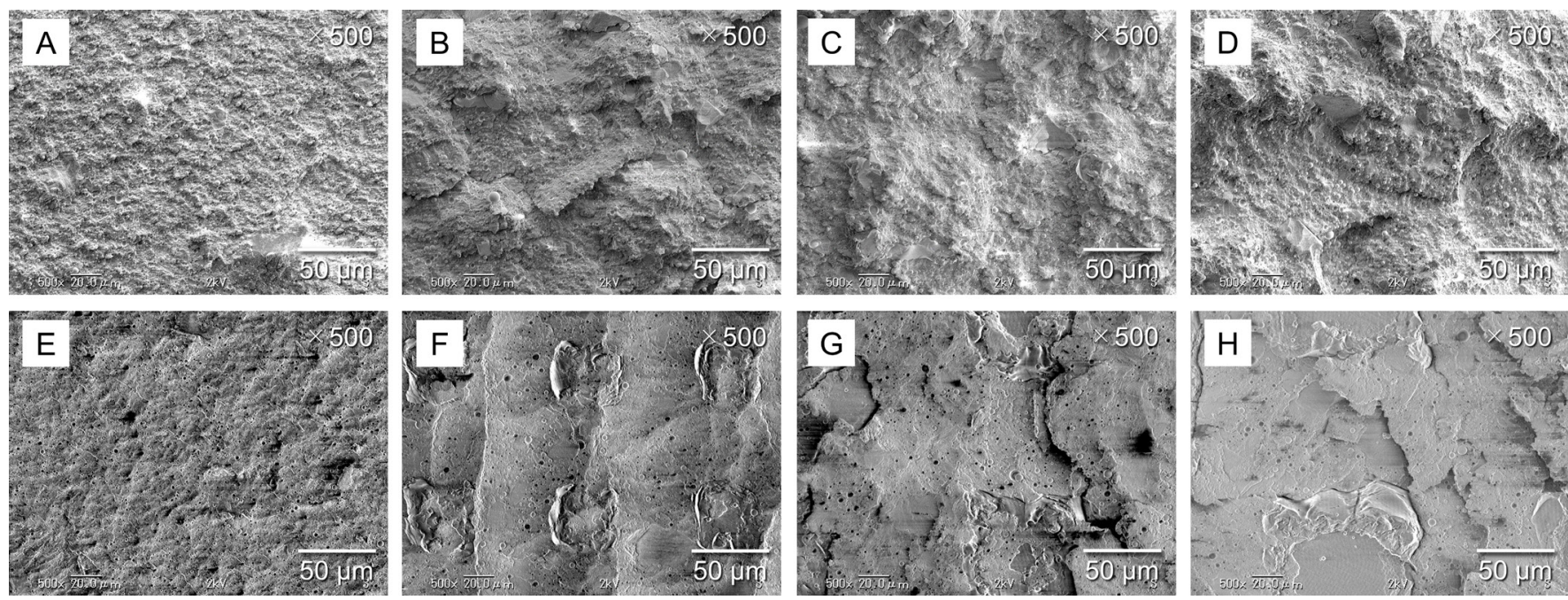

Fig. 7. SEM images of debonded surfaces of Ce-TZP/A samples veneered with indirect composite resin (500×): (A) alumina-blasted specimens and (B-D) specimens with machined microslits with dimensions of 50, 75, and $100 \mu \mathrm{m}$ (MS50, MS75, and MS100, respectively) before thermocycling and (E) alumina-blasted specimens and (F-H) specimens with machined microslits with dimensions of 50, 75, and $100 \mu \mathrm{m}$ (MS50, MS75, and MS100, respectively) after thermocycling.

durability are essential for a positive long-term prognosis.

Several studies reported the need for a particular mechanical retentive structure [14-17]. If a mechanical retention structure is to be formed in zirconia, it should be designed such that the thickness of the frame is not affected significantly while also keeping in mind the veneering space as well as the volume of the tooth preparation. It is impossible to produce microscale structures on zirconia using dental CAD/CAM systems, because the minimum diameter of the milling burs available for these systemsis $0.4-0.6 \mathrm{~mm}$. Furthermore, even if microscale milling burs were to become available, their wear would be severe, because the object material considered in the present study is fully sintered zirconia.

Another approach is laser beam machining, which eliminates the need for burs. There are a few reports on the effects of surface treatment using a $\mathrm{CO}_{2}$, Er:YAG, Nd:YAG, Er,Cr:YSGG, or Nd:YVO 4 laser, on the adhesive strength of dental materials [15-17,26,28,29]. CNC laser machining is commonly used in the industry for the manufacture of injection molds, lettering, and engraving. This processing method allows for stable and controllable machining and can readily produce microscopic surface textures. Therefore, this technology can potentially be used for accurately fabricating microstructures on zirconia frames with a curved surface.

We had previously reported the effect of simple gridded slits (width and pitch: $40 \mu \mathrm{m}$, depth: $25 \mu \mathrm{m}$ ) machined using a $\mathrm{Nd}: \mathrm{YVO}_{4}$ laser on zirconia disks [22]. We had measured the SBS values of porcelain and an indirect composite resin veneered to Y-TZP and Ce-TZP/A samples and had compared the SBS values of specimens with and without the abovementioned microslits. We had found that, although the microslits improved the SBS and adhesion durability of the bonds formed between Y-TZP and the indirect composite resin, the SBS values of the bonds between porcelain and the two types of zirconia or the indirect composite resin and $\mathrm{Ce}-\mathrm{TZP} / \mathrm{A}$ did not increase significantly after microgrid formation. In the present study, in order to elucidate the effects of deeper and wider slits, we fabricated microslit patterns of different dimensions. The minimum laser spot diameter of the laser machine $(30 \mu \mathrm{m})$ and the enlargement of the slit which does not impair the strength of the zirconia frame in clinical use, were considered in setting up the slit dimensions (minimum of $50 \mu \mathrm{m}$ to a maximum of $100 \mu \mathrm{m}$, and $75 \mu \mathrm{m}$ in the middle). Laser processing allowed the uniform and precise formation of microslits in each specimen (Figs. 2 and 3). However, in this study, it took approximately $20 \mathrm{~min}$ to complete 
each specimen because of the careful processing required. It is therefore necessary to shorten the processing time for clinical use.

While Y-TZP is widely used in dentistry, Ce-TZP has superior flexural strength $[5,6]$. Since clinicians choose which one to use as a core material on their respective preferences, therefore, in the present study, both types of zirconia were used to investigate the SBS with veneering materials. Discoloration after laser processing may be caused by oxygen loss owing to high temperatures and fast cooling rates [16]. Therefore, specimens of microslits were heat-treated to restore their white color. Previous studies reported that heat treatment decreased the flexural strength of zirconia $[32,33]$. Although we were concerned about the negative effect of heat treatment on the adhesive strength, no decrease in SBS was observed in the microslit groups investigated in this study.

A reduction in bond strength between composite resin and zirconia is generally expected as a result of exposure to moisture and significant temperature changes in the oral cavity because the water absorption, and coefficient of thermal expansion (CTE) of composite resins are higher than that of zirconia [34]. Therefore, the change in SBS before and after thermocycling was assessed for indirect composite resins. On the other hand, previous studies reported that thermocycling had no influence on the bond strength between porcelain and zirconia $[35,36]$. This may be because the CTE of porcelain is close to that of zirconia and is less susceptible to moisture. Accordingly, thermocycling was not applied for porcelain in this study.

\subsection{Effects of microslits on porcelain veneered to zirconia}

The microslits machined in this study were in the form of a gridded pattern. In our previous study [17], SEM observations obtained after the shear test indicated that the microslits formed on Y-TZP and Ce-TZP/A samples were still completely covered with porcelain. In other words, the porcelain remaining on the zirconia samples was owing to the microslits. However, there were no significant differences between the SBS values of the specimens with and without microslits and we hypothesized that it should be possible to increase the SBS value by modifying the microslits.

As a result, the SBS values in the MS groups of Y-TZP tended to be slightly higher than those in the $\mathrm{AB}$ group, however, there was no statistically significant difference. Similarly, the SBS value in the MS100 group of the Ce-TZP/A increased slightly more than those in the AB group, while no statistical significant difference was found. A statistical significant difference could be found if the sample size increased. In addition, all the specimens in both MS groups showed cohesive failure, suggesting that the microslits maintain the porcelain on the zirconia surface. However, while microslits have shown the possibility of improving the bonding, there is scope for further improvement the SBS.

To improve the SBS, it may be necessary to strengthen the porcelain or modify the retentive structure by using a design that does not allow for the concentration of stress on the upper surface of the microstructure. Several studies have reported that the treatment by CNC-milling [25] or laser $[15,16]$ of zirconia enhances the SBS of the bonds formed with porcelain. Santos et al. [25] evaluated the shear bond strength of porcelain to zirconia substrates modified by CNC-milling process. They found that it is possible to highly enhance the zirconia-porcelain bond strength (even by $\sim 55 \%$ ) by combining surface holes $(\varnothing 300 \mu \mathrm{m})$ in zirconia frameworks and applying of a proper ceramic composite interlayer. Henrique et al. [16] fabricated holes with diameters of 25 and $50 \mu \mathrm{m}$ and depths of approximately 50 and $100 \mu \mathrm{m}$, respectively, in zirconia using a Nd:YAG laser. They concluded that the laser machining of the zirconia surfaces enhanced the SBS between the zirconia and injected feldspathic porcelain by up to $75 \%$. Although the three dimensions of microslits used in this study were similar to those of the above-mentioned holes, we did not observe as significant an increase in the SBS. Abdullah et al. [15] investigated the effects of a $\mathrm{CO}_{2}$ laser treatment on the shear bond strength of zirconia frameworks and veneering ceramics. They found that microholes created using the laser treatment allowed for micromechanical retention and promoted adhesion between the veneering ceramic and zirconia. Thus, the above-described studies found that the bond strength of porcelain and zirconia is improved by CNC-milling or laser treatments and that this is owing to the mechanical interlocking afforded by the machined holes, which improves the bonds formed. This also explains why zirconia with spherical pores bonds strongly with porcelain [14].

We did not observe any improvements in the SBS between porcelain and zirconia after the formation of the microslits possibly owing to the presence of a liner. In this study, a liner was applied because the experiments were designed to minimize the effects of the color of the abutment teeth on the shade of the veneering porcelain. We also expected that the liner would easily penetrate the microslit because of its paste-like consistency. However, Fischer et al. [21] evaluated the bond strength of different veneering ceramics with $\mathrm{Ce}-\mathrm{TZP} / \mathrm{A}$ samples treated with a liner as well as with those not subjected to the treatment. They found that the application of the liner resulted in a small but significant decrease in the shear bond strength. Mosharraf et al. [20] evaluate of the effect of different surface treatments and types of zirconia (white or colored) on the SBS of zirconia and veneering porcelain. They reported that liner application reduced the mean SBS in white zirconia. On the other hand, a previous study reported that liner application on pre-sintered zirconia enhanced the SBS [37]. Other researchers found that the novel experimental liner containing $3.0 \mathrm{wt} \%$ zirconia significantly enhanced the zirconia/porcelain interfacial bonding [38]. Therefore, liners have varying effects on adhesion depending on their use and ingredients, and further investigations are required to confirm whether the use of a line affects the bonds formed between porcelain and zirconia with machined microslits.

\subsection{Effects of microslits on indirect composite resin veneered to zirconia}

Several researchers have measured the SBS between zirconia and indirect composite resins after thermocycling and found that the application of the appropriate priming agent and the roughening of the zirconia surface improve bond durability [7,22]. In our previous study [17], we also found that microslits (width and pitch: $40 \mu \mathrm{m}$, depth: $25 \mu \mathrm{m}$ ) are effective in improving the bond strength between indirect composite resins and zirconia. Even after 20,000 thermocycles, the SBS of the specimens with microslits remained unchanged. Thus, the specimens with the microslits could withstand more severe conditions than those in the untreated group. However, the SBS of the Ce-TZP/A specimens with microslits after thermocycling was significantly lower than that of the specimens subjected to alumina blasting. Therefore, in this study, the dimensions of the microslits were modified to afford stronger mechanical interlocking.

Previous studies have suggested that 10-methacryloyloxydecyl dihydrogen phosphate (MDP) consisting of phosphate monomers is effective in improving the bond strength between indirect composite resin and zirconia $[39,40]$. Therefore, in this study, a primer including MDP was used in the clinical application of chemical and mechanical bonding of indirect composite resin and zirconia.

In the present study, before thermocycling, there was no significant difference between the $\mathrm{AB}$ groups and the MS groups in both type of zirconia. The fact that the $\mathrm{AB}$ groups showed bond strength comparable to that of the MS groups may be attributable to the interaction between the surface roughening and the primer. Shimoe et al. [8] evaluated the influence of air-abrasion on the SBS between indirect composites and zirconia and suggested that air-abrasion is highly effective in improving the bonding. The SBS values of the alumina group in the present experiments (Y-TZP: $28.9 \mathrm{MPa}$; Ce-TZP/A: $25.6 \mathrm{MPa}$ ) are relatively close to their reported values. However, the SBS values of $\mathrm{AB}$ groups in both types of zirconia showed a significant decrease after thermocycling. The MS groups showed no decrease in SBS after thermocycling, and the values were comparable to the SBS of the conventional metal-composite combination $[18,41]$.

There was a tendency for the SBS to increase after thermocycling in the microslit groups, except for the MS 75 group of Y-TZP, although it was not statistically significant. Similarly, the studies that evaluated the bond strength of resin composite to metal reported an increase in the shear bond strength following thermocycling $[18,42]$. These may be attributable to post-curing polymerization caused by heating during storage or thermocycling. Previous studies have reported that increasing the temperature of composite resin can significantly influences resin 
polymerization and could lead to improved properties of composite resin $[43,44]$. However, regardless of the amount of polymerization by heating, if the composite resin and core materials are not strongly bonded, the SBS values after thermocycle decrease as observed in alumina-blasted groups in this study. It can therefore be inferred that microslits with dimensions of $50-100 \mu \mathrm{m}$ are effective in improving the adhesion strength and durability in the case of the investigated indirect composite resin and this is true for both Y-TZP and Ce-TZP/A.

The surface treatment by laser irradiation can also be used for cementation procedures [27], because the accurate machining of the inner surface of the zirconia coping is possible using a $\mathrm{Nd}$ : $\mathrm{YVO}_{4}$ laser [29]. However, other studies suggested that surface treatment by dental laser on zirconia decrease or did not improve the bond strength compared with alumina blasting $[9,23]$. Those studies used a dental laser to roughen the surface for chairside use. In contrast, we used an industrial laser machine to fabricate the microslits of uniform dimensions on the zirconia surface. In particular, the fact that the slit was lattice-shaped may be the reason for the positive results, unlike in the aforementioned studies. Thus, the microstructure design used in this study would be suitable for the inner surfaces of zirconia restorations for effectively improving bonding with resin cements.

There was no significant difference between the groups with respect to the microslits of different dimensions. Therefore, within the range of dimensions used in this study, even if the microslits were fabricated deeper and wider, the bond strength between the indirect composite resin and zirconia would not be affected. To improve the retentive structure for effective bonding, complex fabrication, such as having undercuts as well as size may be necessary.

Overall, the microslits formed in the zirconia disks using CNC laser machining improved the adhesive strength of the bonds formed with the indirect composite resin. The fact that the effectiveness of the microslit for bonding to porcelain was less than that for bonding the indirect composite resin in terms of SBS may be due to the brittleness of the porcelain. Tsitrou et al. [45] measure the brittleness index (BI) and the chipping factor (CF) of a range of dental materials to assess their relationship and found that the CF of porcelain was significantly higher than that of composite resin. They further concluded that as the BI of a material increases so does the $\mathrm{CF}$. Although the flexural strength of the porcelain and the composite resin for the same products as in this study differed slightly at approximately 80 $90 \mathrm{MPa}$ [46,47], because the porcelain is generally more brittle than the composite resin, it is possible that the porcelain in the microslit did not withstand the shear forces as much as the investigated indirect composite resin and was fractured.

The precision of CNC laser processing was confirmed in this study, although the effect in the case of porcelain was limited. Thus, the noncontact processing of zirconia through $\mathrm{CNC}$ laser irradiation should be developed further. Moreover, the results of this study confirm that the design of the mechanical retentive structure formed in zirconia should be based on the properties of the veneering material to be used. A recent study reported that laser structuring to enhance the bond strength results in the formation of microcracks [16]. Although laser processing in our study may also have caused microcracks, their presence is expected to be within a localized area on the zirconia surface, accordingly further investigation should be carried out to compare the strength of zirconia after CNC laser processing and with the strength after alumina blasting or dental laser processing.

A limitation of this study is that the design of the zirconia specimens used was simplified for the shear test and therefore did not completely follow the frame design used in clinical practice. Another limitation is that, in clinical practice, more colored materials are layered on zirconia and fired or polymerized more times than in this experiment, and the veneered materials are contoured and polished by burs or points, however the present experiment did not fully simulate those clinical routines.

\section{Conclusion}

Within the limitation of the study, the following conclusions can be drawn:

(1) The slit-shaped microstructure with dimensions of 50-100 $\mu \mathrm{m}$ formed on two types of zirconia (Y-TZP and Ce-TZP/A) did not enhance the shear bond strength in the case of porcelain.

(2) After thermocycling, the strength of the bonds formed between the investigated indirect composite resin and the Y-TZP and Ce-TZP/A specimens with the microslits were significantly higher than those of the specimens in the alumina-blasted group.

(3) With respect to the bonding of the indirect composite resin to the two types of zirconia, there was no significant difference between the bond strengths of the specimens with the microslits of different dimensions $(50,75$, and $100 \mu \mathrm{m})$.

\section{Acknowledgements}

This study was supported in part by a Grant-in-Aid for Scientific Research (Grant Number C 16K11593; 2016-2018) from the Japan Society for the Promotion of Science (JSPS).

\section{Conflicts of Competing interest}

The authors declare that they have no conflicts of interest.

\section{References}

[1] Butler EP. Transformation-toughened zirconia ceramics. Mater Sci Technol 1985;1:417-32. https://doi.org/10.1179/mst.1985.1.6.417.

[2] Nassary Zadeh P, Lümkemann N, Sener B, Eichberger M, Stawarczyk B. Flexural strength, fracture toughness, and translucency of cubic/tetragonal zirconia materials. J Prosthet Dent 2018;120:948-54. https://doi.org/10.1016/ j.prosdent.2017.12.021.

[3] Harada K, Shinya A, Yokoyama D, Shinya A. Effect of loading conditions on the fracture toughness of zirconia. J Prosthodont Res 2013;57:82-7. https://doi. org/10.1016/j.jpor.2013.01.005.

[4] Piconi C, Maccauro G. Zirconia as a ceramic biomaterial. Biomaterials 1999;20:1-25. https://doi.org/10.1016/S0142-9612(98)00010-6.

[5] Güngör MB, Yilmaz H, Aydin C, Nemli SK, Bal BT, Tiraş T. Biaxial flexural strength and phase transformation of Ce-TZP/A12O3 and Y-TZP core materials after thermocycling and mechanical loading. J Adv Prosthodont 2014;6:224-32. https://doi.org/10.4047/jap.2014.6.3.224.

[6] Ban S, Sato H, Suehiro Y, Nakanishi H, Nawa M. Biaxial flexure strength and low temperature degradation of $\mathrm{Ce}-\mathrm{TZP} / \mathrm{Al} 2 \mathrm{O} 3$ nanocomposite and Y-TZP as dental restoratives. J Biomed Mater Res - Part B Appl Biomater 2008;87:492-8. https://doi.org/10.1002/jbm.b.31131.

[7] Komine F, Kobayashi K, Saito A, Fushiki R, Koizumi H, Matsumura H. Shear bond strength between an indirect composite veneering material and zirconia ceramics after thermocycling. J Oral Sci 2009;51:629-34. https://doi. org/10.2334/josnusd.51.629.

[8] Shimoe S, Peng T-Y, Otaku M, Tsumura N, Iwaguro S, Satoda T. Influence of various airborne-particle abrasion conditions on bonding between zirconia ceramics and an indirect composite resin material. J Prosthet Dent 2019;122:491.e1-e9. https://doi.org/10.1016/j.prosdent.2019.08.016.

[9] Lee SJ, Cheong CW, Wright RF, Chang BM. Bond strength of the porcelain repair system to all-ceramic copings and porcelain. J Prosthodont 2014;23:1126. https://doi.org/10.1111/jopr.12064.

[10] Komine F, Koizuka M, Fushiki R, Iwasaki T, Kubochi K, Matsumura H. Effect of various surface preparations on bond strength of a gingiva-colored indirect composite to zirconia framework for implant-supported prostheses. Dent Mater J 2015;34:402-9. https://doi.org/10.4012/dmj.2014-360.

[11] Esquivel J, Lawson NC, Kee E, Bruggers K, Blatz MB. Wear of resin teeth opposing zirconia. J Prosthet Dent 2020;124:488-93. https://doi.org/10.1016/ j.prosdent.2019.11.004.

[12] Stober T, Bermejo JL, Schwindling FS, Schmitter M. Clinical assessment of enamel wear caused by monolithic zirconia crowns. J Oral Rehabil 2016;43:621-9. https://doi.org/10.1111/joor.12409.

[13] Jang YS, Nguyen TDT, Ko YH, Lee DW, Baik BJ, Lee MH, et al. In vitro wear behavior between enamel cusp and three aesthetic restorative materials: Zirconia, porcelain, and composite resin. J Adv Prosthodont 2019;11:7-15. https://doi.org/10.4047/jap.2019.11.1.7.

[14] Nakamura T, Sugano T, Usami H, Wakabayashi K, Ohnishi H, Sekino T, et al. Shear bond strength of veneering porcelain to porous zirconia. Dent Mater J 2014;33:220-5. https://doi.org/10.4012/dmj.2013-191.

[15] Abdullah AO, Yu H, Pollington S, Muhammed FK, Xudong S, Liu Y. Effect of repeated laser surface treatments on shear bond strength between zirconia and veneering ceramic. J Prosthet Dent 2020;123:338.e1-e6. https://doi. org/10.1016/j.prosdent.2019.10.007. 
[16] Henriques B, Fabris D, Souza JCM, Silva FS, Carvalho Ó, Fredel MC, et al. Bond strength enhancement of zirconia-porcelain interfaces via $\mathrm{Nd}: \mathrm{YAG}$ laser surface structuring. J Mech Behav Biomed Mater 2018;81:161-7. https://doi. org/10.1016/j.jmbbm.2018.02.031.

[17] Iwaguro S, Shimoe S, Hirata I, Murayama T, Satoda T. Effect of microslit retention on the bond strength of zirconia to dental materials. Dent Mater J 2019;38:1043-52. https://doi.org/10.4012/dmj.2018-351.

[18] Orchard NA, Howlett JA, Davies EH, Pearson GJ. Adhesive composite resins for artificial teeth: a laboratory investigation of bond strength to a cobaltchromium alloy. Biomaterials 1997;18:935-8. https://doi.org/10.1016/S01429612(97)00023-9.

[19] Shue SL, Nicholls JI, Townsend JD. The effect of metal retentive designs on resin veneer retention. J Prosthet Dent 1987;58:297-305. https://doi. org/10.1016/0022-3913(87)90044-8.

[20] Mosharraf R, Rismanchian M, Savabi O, Ashtiani AH. Influence of surface modification techniques on shear bond strength between different zirconia cores and veneering ceramics. J Adv Prosthodont 2011;3:221-8. https://doi. org/10.4047/jap.2011.3.4.221.

[21] Fischer J, Stawarczyk B, Sailer I, Hämmerle CHF. Shear bond strength between veneering ceramics and ceria-stabilized zirconia/alumina. J Prosthet Dent 2010;103:267-74. https://doi.org/10.1016/S0022-3913(10)60056-X.

[22] Shimoe S, Tanoue N, Kusano K, Okazaki M, Satoda T. Influence of air-abrasion and subsequent heat treatment on bonding between zirconia framework material and indirect composites. Dent Mater J 2012;31:751-7. https://doi.org/10.4012/ dmj.2012-077.

[23] Mahmoodi N, Hooshmand T, Heidari S, Khoshro K. Effect of sandblasting, silica coating, and laser treatment on the microtensile bond strength of a dental zirconia ceramic to resin cements. Lasers Med Sci 2016;31:205-11. https://doi. org/10.1007/s10103-015-1848-9.

[24] Xie H, Chen C, Dai W, Chen G, Zhang F. In vitro short-term bonding performance of zirconia treated with hot acid etching and primer conditioning etching and primer conditioning. Dent Mater J 2013;32:928-38. https://doi. org/10.4012/dmj.2013-010.

[25] Santos RLP, Silva FS, Nascimento RM, Souza JCM, Motta F V., Carvalho $\mathrm{O}$, et al. Shear bond strength of veneering porcelain to zirconia: Effect of surface treatment by $\mathrm{CNC}$-milling and composite layer deposition on zirconia. J Mech Behav Biomed Mater 2016;60:547-56. https://doi.org/10.1016/ j.jmbbm.2016.03.015.

[26] Noda M, Okuda Y, Tsuruki J, Minesaki Y, Takenouchi Y, Ban S. Surface damages of zirconia by Nd:YAG dental laser irradiation. Dent Mater J 2010;29:536-41. https://doi.org/10.4012/dmj.2009-127.

[27] Liu L, Liu S, Song X. Effect of Nd : YAG laser irradiation on surface properties and bond strength of zirconia ceramics. Laser Med Sci 2015:627-34. https:// doi.org/10.1007/s10103-013-1381-7.

[28] Tokar E, Polat S, Ozturk C. Repair bond strength of composite to Er,Cr:YSGG laser irradiated zirconia and porcelain surfaces. Biomed J 2019;42:193-9. https://doi.org/10.1016/j.bj.2019.02.001.

[29] Kazama-Koide M, Ohkuma K, Ogura H, Miyagawa Y. A new method for fabricating zirconia copings using a Nd:YVO4 nanosecond laser. Dent Mater J 2014;33:422-9. https://doi.org/10.4012/dmj.2013-348.

[30] Shimoe S, Peng T, Wakabayashi Y, Takenaka H, Iwaguro S, Kaku M. Lasermilled microslits improve the bonding strength of acrylic resin to zirconia ceramics. Polymers 2020;12:817. https://doi.org/10.3390/polym12040817.

[31] Faeghinejad M, Proussaefs P, AlHelal A, Lozada J. The CAD/CAM compound prosthesis: digital workflow for fabricating cement-retained zirconia prosthesis over screw-retained milled titanium bars. Int J Periodontics Restorative Dent 2019;39:39-47. https://doi.org/10.11607/prd.3409.
[32] Sato H, Yamada K, Pezzotti G, Nawa M, Ban S. Mechanical properties of dental zirconia ceramics changed with sandblasting and heat treatment. Dent Mater J 2008;27:408-14. https://doi.org/10.4012/dmj.27.408.

[33] Passos SP, Linke B, Major PW, Nychka JA. The effect of air-abrasion and heat treatment on the fracture behavior of Y-TZP. Dent Mater 2015;31:1011-21. https://doi.org/10.1016/j.dental.2015.05.008.

[34] Rajan G, Shouha P, Ellakwa A, Bhowmik K, Xi J, Prusty G. Evaluation of the physical properties of dental resin composites using optical fiber sensing technology. Dent Mater 2016;32:1113-23. https://doi.org/10.1016/ j.dental.2016.06.015.

[35] Guess PC, Kuliš A, Witkowski S, Wolkewitz M, Zhang Y, Strub JR. Shear bond strengths between different zirconia cores and veneering ceramics and their susceptibility to thermocycling. Dent Mater 2008;24:1556-67. https://doi. org/10.1016/j.dental.2008.03.028.

[36] Ghaffari T, Moslehifard E, Motiei M. Effect of thermal and mechanical cycles on shear bond strength of zirconia core to porcelain veneer under different surface treatments. J Dent Res Dent Clin Dent Prospects 2019;13:227-33. https://doi.org/10.15171/joddd.2019.035.

[37] Kirmali O, Akin H, Ozdemir AK. Shear bond strength of veneering ceramic to zirconia core after different surface treatments. Photomed Laser Surg 2013;31:261-8. https://doi.org/10.1089/pho.2013.3487.

[38] Lee HS, Kwon TY. The application of a novel ceramic liner improves bonding between zirconia and veneering porcelain. Materials 2017;10:1-12. https://doi. org/10.3390/ma10091023.

[39] Akgungor G, Sen D, Aydin M. Influence of different surface treatments on the short-term bond strength and durability between a zirconia post and a composite resin core material. J Prosthet Dent 2008;99:388-99. https://doi.org/10.1016/ S0022-3913(08)60088-8

[40] Lima RBW, Barreto SC, Alfrisany NM, Porto TS, De Souza GM, De Goes MF. Effect of silane and MDP-based primers on physico-chemical properties of zirconia and its bond strength to resin cement. Dent Mater 2019;35:1557-67. https://doi.org/10.1016/j.dental.2019.07.008.

[41] Shimoe S, Tanoue N, Yanagida H, Atsuta M, Koizumi H, Matsumura H. Comparative strength of metal-ceramic and metal-composite bonds after extended thermocycling. J Oral Rehabil 2004;31:689-94. https://doi. org/10.1111/j.1365-2842.2004.01391.x.

[42] Ohkubo C, Kono H, Tanaka Y, Watanabe I. Shear bond strength of resin composite to magnetic Fe-Pt alloy. J Prosthet Dent 2005;93:478-82. https://doi. org/10.1016/j.prosdent.2005.01.015.

[43] Par M, Gamulin O, Marovic D, Klaric E, Tarle Z. Effect of temperature on postcure polymerization of bulk-fill composites. J Dent 2014;42:1255-60. https:// doi.org/10.1016/j.jdent.2014.08.004.

[44] Trujillo M, Newman SM, Stansbury JW. Use of near-IR to monitor the influence of external heating on dental composite photopolymerization. Dent Mater 2004;20:766-77. https://doi.org/10.1016/i.dental.2004.02.003.

[45] Tsitrou EA, Northeast SE, van Noort R. Brittleness index of machinable dental materials and its relation to the marginal chipping factor. J Dent 2007;35:897902. https://doi.org/10.1016/j.jdent.2007.07.002.

[46] Fischer J, Stawarczyk B, Hämmerle CHF. Flexural strength of veneering ceramics for zirconia. J Dent 2008;36:316-21. https://doi.org/10.1016/ j.jdent.2008.01.017.

[47] Scribante A, Bollardi M, Chiesa M, Poggio C, Colombo M. Flexural properties and elastic modulus of different esthetic restorative materials: Evaluation after exposure to acidic drink. Biomed Res Int 2019;2019:1-8. https://doi. org/10.1155/2019/5109481.

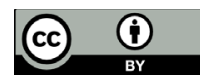

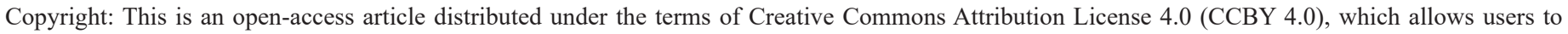
distribute and copy the material in any format so long as attribution is given to the author(s). 